\title{
A Thermodynamic Modeling Approach for Dynamic Softening in Glassy Amorphous Polymers
}

\author{
Rui Xiao ${ }^{\mathrm{a}}$, Thao D. Nguyen ${ }^{\mathrm{b}}$ \\ ${ }^{a}$ Institute of Soft Matter Mechanics, College of Mechanics and Materials, Hohai University, Nanjing, Jiangsu 210098, China \\ ${ }^{b}$ Department of Mechanical Engineering, The Johns Hopkins University, Baltimore, MD 21218, USA
}

\begin{abstract}
Strain softening is highly dependent on the thermo-mechanical history. The degree of post-yield strain softening increases with the aging time, which is dependent on the aging temperature, and decreases with plastic strain. In this paper, we present two approaches to describe the strain softening. The first approach is an extension of the widely applied dynamic softening method that evolves the yield strength with the effective plastic strain rate. Though this approach can quantitatively capture the main experimental observations, it is empirical and does not capture the physical mechanism behind aging and strain softening. The second approach couples inelastic deformation with an evolution of the nonequilibrium state. The approach is based on the effective temperature nonequilibrium thermodynamic framework that introduces a thermodynamic state variable, the effective temperature to characterize the nonequilibrium state. The temperature-dependent evolution of the effective temperature describes the glass transition of amorphous polymers. We show that the physical aging and dynamic softening arise from the evolution of the effective temperature towards equilibrium and with plastic deformation towards a steady-state value away from equilibrium.
\end{abstract}

Keywords: Physical aging, mechanical rejuvenation, strain softening

\section{Introduction}

The stress response of glassy polymers typically exhibits four different regions: a linear increase in stress with strain (elastic region), a drop in stress with strain (post-yield strain softening), a nearly constant stress with strain (steady state draw), and an increase in stress with strain (orientation hardening). Understanding the strain softening of glassy amorphous polymers is crucial in many applications, because softening induces strain localization that leads to failure of polymers $[1,2]$. The post-yield softening behavior is strongly dependent on temperature, strain rate and thermomechanical loading history [3-6]. When glassy polymers are annealed, the yield strength increases with annealing time, known as physical aging [3,6-8]. In contrast, the steady state flow stress is independent of previous thermal history [6]. The post-yield stress drop can be eliminated by large plastic pre-deformation such as in cold rolling and cold drawing [5]. This phenomena is opposite to physical aging, and is referred as mechanical rejuvenation by Struik [4]. The softening recovers with time indicating a renewed physical aging after cessation of plastic deformation.

To describe this complex dynamic softening behavior, numerous constitutive models have been developed that used an internal variable to describe the nonequilibrium structure and its effect on the yield strengh [9-16]. Boyce et al. [9] proposed the evolution of the yield strength to a steady state value with plastic strain rate, which has been widely employed to model the strain softening. Hasan and Boyce [10] developed an evolution equation for the number of shear transformation sites to describe the strain softening behaviors of quenched and annealed specimens of poly(methyl

Email address: rxiao@hhu.edu.cn (Rui Xiao) 
methacrylate), while Klompen et al. [11] developed an evolution equation for a phenomenological state variable to describe the softening behaviors of polycarbonate. Buckley et al. [12] applied fictive temperature $\left(T_{f}\right)$ concept of Tool [17] to model the strain softening. They proposed a phenomenological evolution equation for the fictive temperature that increases with the effective viscous strain rate to represent the mechanical rejuvenation.

Though these models have achieved considerable success in modeling the post-yield strain softening, they cannot capture certain important features observed in experiments. For example, the internal variable approach of Boyce and coworkers cannot capture the recovery of the yield strength after cessation of plastic deformation. In this letter, we present a new thermodynamic approach to model the effect of physical aging and plastic deformation on the post-yield strain softening behavior and compare it to the widely used internal variable softening model of Boyce et al. [9], which we extend to include static recovery of the yield strength [18]. The approach is based on the effective temperature theory, which introduces the effective temperature as a thermodynamic state variable for the nonequilibrium configurational structure [19-22]. The central assumption of the effective temperature theory is that the total degrees of freedom of amorphous polymers are composed of vibrational degrees of freedom characterized by the current temperature, and configurational degrees of freedom characterized by the effective temperature. The effective temperature theory has been applied successfully to describe amorphous metals and granular matter [2024]. The ability of the effective temperature model to describe softening has been demonstrated by STZ theory and discussed in $[22,25]$.

Here, we show that the effective temperature approach can be used to describe the yield and dynamic softening response of polymers. For amorphous polymers, we develop models for the viscoelastic internal energy and entropy density of the vibrational and configurational subsystems, based on the entropy elasticity model of Chadwick [26]. We introduce a distribution of viscous deformation and effective temperatures, along with discrete spectrums of structural and stress relaxation times, to accurately represent the time-dependent stress response and physical aging. Finally, we develop a model for the stress and structural relaxation times that depends on temperature and the nonequilibrium structure through the configurational entropy. In the model, the increase in the yield stress with physical aging occurs because the nonequilibrium structure evolves towards a more ordered state, decreasing the molecular mobility and retarding plastic flow. When deformed, part of the plastic work is stored in configurational rearrangements to a more disordered state, which increases the molecular mobility, promoting plastic flow and producing strain softening [27]. This is one of fundamental differences between the effective temperature thermomechanical theory and classic thermomechanical theory. In the classic thermomechanical theory, all the plastic power is transferred into regular heat and entropy, which is not consistent with the following experimental observations[12, 28]. The steady-state flow stress is obtained when the rejuvenation effect balances the aging effect. In the following sections, we show that the effective temperature approach can capture more accurately the time-dependent and temperature-dependent effect of physical aging and mechanical rejuvenation on dynamic softening, and more generally on the stress response, than the internal variable approach.

\section{Experimental observation}

We performed a series of uniaxial compression experiments on an acrylate-based random copolymer [29] to characterize the rate-dependence and time-dependence of the post-yield softening response. The polymer has a $10 \%$ cross-linker density and exhibits a $T_{\mathrm{g}}$ at $36^{\circ} \mathrm{C}$. We used cylindrical specimens $6.0 \mathrm{~cm}$ in diameter and height. The specimens were first thermally rejuvenated at $70^{\circ} \mathrm{C}$ for 30 minutes, cooled to $30^{\circ} \mathrm{C}, 20^{\circ} \mathrm{C}, 10^{\circ} \mathrm{C}$ or $0^{\circ} \mathrm{C}$ at $3^{\circ} \mathrm{C} / \mathrm{min}$, and annealed for 30 minutes. The specimens were compressed to $30 \%$ engineering strain at an engineering strain rate $0.0001 / \mathrm{s}, 0.001 / \mathrm{s}$ or 0.01/s using an MTS Insight 5 electromechanical testing system. As shown in Fig. 1, the stress response showed the typical features of yield, a post-yield stress drop to a steady-state draw stress. The yield strength, post-yield stress drop, and flow stress increased with increased strain rate and decreased temperature.

We next investigated the effect of physical aging and plastic pre-deformation on the stress response by performing a series of load-unload-reload tests in compression. After heating and equilibrating at $70^{\circ} \mathrm{C}$ for $30 \mathrm{mins}$, the specimens were cooled to $20^{\circ} \mathrm{C}$ at $3^{\circ} \mathrm{C} / \mathrm{min}$, and annealed for 30 minutes, 1 day or 14 days in an incubator (Torrey Pines Scientific, Inc, CA,USA) at $20^{\circ} \mathrm{C}$. The specimens were compressed in the MTS with an environmental thermal chamber 


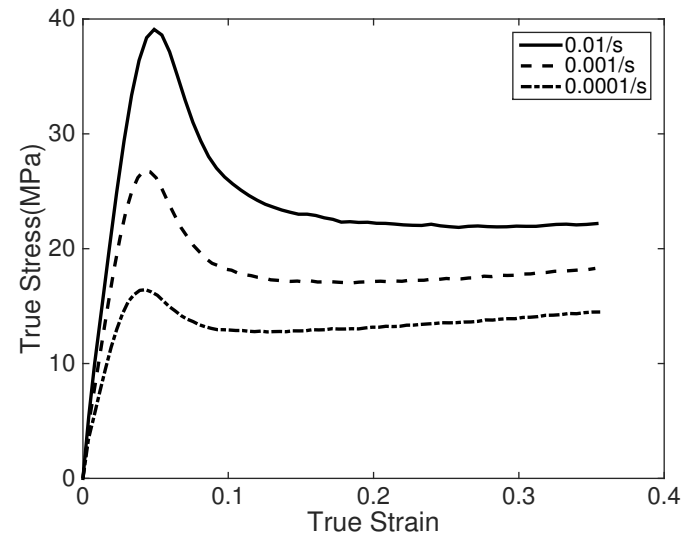

(a)

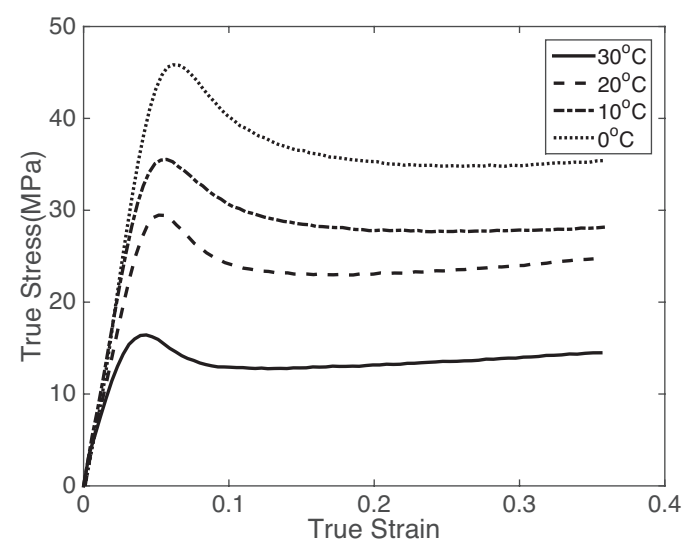

(b)

Figure 1: Experimental results of uniaxial compression response of acrylate-based copolymer (a) at $30^{\circ} \mathrm{C}$ (b) at $0.0001 / \mathrm{s}$

(Thermcraft, Inc. NC, USA) set at $20^{\circ} \mathrm{C}$ at a strain rate of $0.001 / \mathrm{s}$ to $30 \%$ engineering strain. The specimens were annealed again at the same temperature for 1 minute, 10 minutes, 1 day or 14 days before being compressed to an additional $15 \%$ strain at $0.001 / \mathrm{s}$. For the long term storage ( 1 day or 14 days), the specimens were placed in the incubator. Figure 2 shows the experimental results of loading-unloading-reloading tests. The yield strength and the post-yield stress drop increased with annealing period due to physical aging. After around $15 \%$ deformation, the flow stress decreased to the same level in spite of different thermal and mechanical history, indicating the large mechanical deformation rejuvenated the polymer structure to the same state. Considerable strain recovery occurred during the second annealing period because the annealing temperature is close to the onset $T_{g}=36{ }^{\circ} \mathrm{C}$. All the above tests have been repeated at least twice. The results showed good repeatability. In all the tests, the difference of stress between the two repeated tests is smaller than $1 \mathrm{MPa}$.

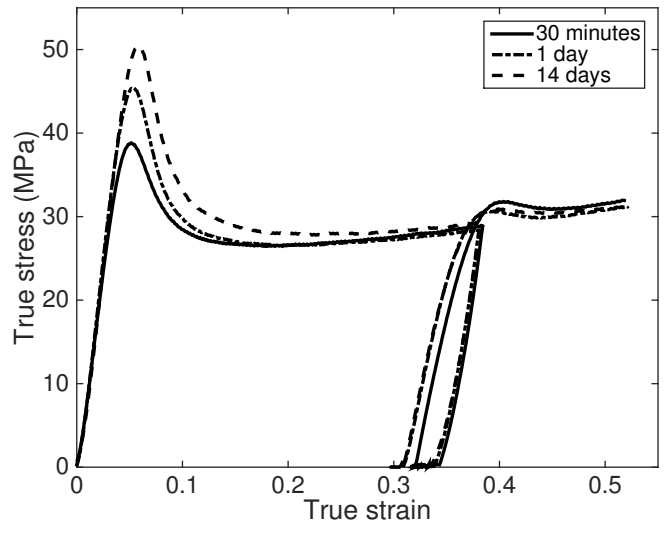

(a)

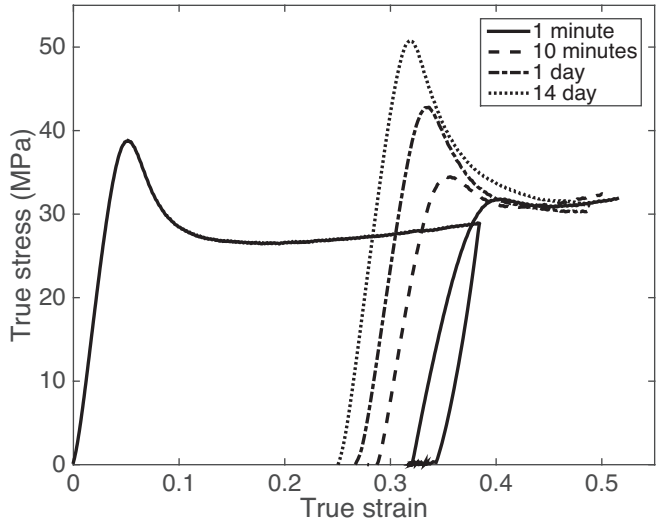

(b)

Figure 2: Experimental results of uniaxial compression response of acrylate-based copolymer for two load-unload cycles at $0.001 / \mathrm{s}$ strain rate (a) different annealing times prior to the first cycle and (b) different annealing time prior to the second cycle

\section{Constitutive model}

We provide two approaches to model dynamic softening: the effective temperature approach [30] and the internal variable approach, which is an extension of the constitutive model of Boyce et al. [9] and Xiao et al. [29]. In the 
following, we describe the constitutive relations of each approach.

\subsection{Effective temperature approach}

Here, we summarize the main equations of the Xiao and Nguyen effective temperature theory for the thermomechanical behavior of amorphous polymers. A detailed derivation of the effective temperature theory can be found in Xiao and Nguyen. [30] from equations 6-38. We begin by defining the kinematics of finite deformation. To model viscous deformation, the deformation gradient $\mathbf{F}$ is decomposed into $N$ parallel elastic and viscous parts as, $\mathbf{F}=\mathbf{F}_{j}^{\mathrm{e}} \mathbf{F}_{j}^{\mathrm{v}}$, $j=1 \ldots N$. The isochoric part of deformation gradient is given by $\overline{\mathbf{F}}=J^{-1 / 3} \mathbf{F}$ and $\overline{\mathbf{F}}_{j}^{\mathrm{e}}=J_{j}^{\mathrm{e}-1 / 3} \mathbf{F}_{j}^{\mathrm{e}}$, where $J=\operatorname{det}(\mathbf{F})$ and $J_{j}^{\mathrm{e}}=\operatorname{det}\left(\mathbf{F}_{j}^{\mathrm{e}}\right)$. The total and elastic isochoric left Cauchy deformation tensor is defined as $\overline{\mathbf{b}}=\overline{\mathbf{F}} \overline{\mathbf{F}}^{\mathrm{T}}$ and $\overline{\mathbf{b}}_{j}^{\mathrm{e}}=\overline{\mathbf{F}}_{j}^{\mathrm{e}} \overline{\mathbf{F}}_{j}^{\mathrm{eT}}$. The corresponding right Cauchy deformation tensor is defined as $\overline{\mathbf{C}}=\overline{\mathbf{F}}^{\mathrm{T}} \overline{\mathbf{F}}$ and $\overline{\mathbf{C}}_{j}^{\mathrm{e}}=\overline{\mathbf{F}}_{j}^{\mathrm{eT}} \overline{\mathbf{F}}_{j}^{\mathrm{e}}$. We also define the velocity gradient of total and viscous deformation tensor as, $\mathbf{L}=\dot{\mathbf{F}} \mathbf{F}^{-1}$ and $\mathbf{L}_{j}^{\mathrm{v}}=\dot{\mathbf{F}}_{j}^{\mathrm{v}} \mathbf{F}_{j}^{\mathrm{v}-1}$. The symmetric total and viscous velocity gradient can be further defined as $\mathbf{D}=\left(\mathbf{L}+\mathbf{L}^{\mathrm{T}}\right) / 2$ and $\mathbf{D}_{j}^{\mathrm{v}}=\left(\mathbf{L}_{j}^{\mathrm{v}}+\mathbf{L}_{j}^{\mathrm{vT}}\right) / 2$.

The central assumption of the effective temperature theory is that the polymer is composed of a fast relaxation kinetic subsystem and slow relaxation configurational subsystems. The internal energy $e$, entropy $\eta$, heat flux $\mathbf{Q}$ and entropy flux $\mathbf{H}$ are correspondingly split into a kinetic part and multiple configurational parts. The effective temperature $T_{e_{i}}$ and the configurational entropy $\eta_{i}^{c}$ are thermodynamic conjugate pairs that are analogous to the temperature and kinetic entropy. Thus, the Helmholtz free energy density $\Psi$ is defined as [19],

$$
\Psi=\underbrace{e^{k}-T \eta^{k}}_{\Psi^{k}}+\sum_{i}^{P} \underbrace{e_{i}^{c}-T_{e_{i}} \eta_{i}^{c}}_{\Psi_{i}^{c}},
$$

where the superscript $k$ refers the kinetic component, and the superscript $c$ together with subscript $i$ refers the ith configurational component.

We use the following explicit functions to describe the Helmholtz free energy density,

$$
\begin{aligned}
& \Psi^{k}=\sum_{j}^{N} \frac{(1-a) \mu_{j}^{\mathrm{neq}}}{2}\left(\operatorname{tr}\left(\overline{\mathbf{C}_{j}^{\mathrm{e}}}\right)-3\right)+\frac{\kappa}{4}\left(J^{2}-2 \log J-1\right)+c_{g}\left(T-T_{0}\right)-c_{g} T \ln \frac{T}{T_{0}}, \\
& \Psi_{i}^{c}=\phi_{i} \frac{T_{e_{i}}}{T_{0}} \frac{\mu^{\mathrm{eq}}}{2}(\operatorname{tr}(\overline{\mathbf{C}})-3)+\phi_{i} \sum_{j}^{N} \frac{a \mu_{j}^{\mathrm{neq}}}{2}\left(\operatorname{tr}\left(\overline{\mathbf{C}}_{j}^{\mathrm{e}}\right)-3\right)+\phi_{i} \Delta c\left(T_{e_{i}}-T_{0}\right)-\phi_{i} \Delta c T_{e_{i}} \ln \frac{T_{e_{i}}}{T_{2}},
\end{aligned}
$$

where $\mu^{\mathrm{eq}}$ is the equilibrium shear modulus, $\mu_{j}^{\text {neq }}$ are the nonequilibrium shear moduli of the viscoelastic spectrum, $\kappa$ is the bulk modulus, $c_{g}$ is the heat capacity of kinetic subsystem, $\Delta c$ is heat capacity of configurational subsystems, $\phi_{i}$ describes the spectrum of the configurational subsystems, where $\sum_{i}^{P} \phi_{i}=1$, and $a$ is the partition parameter of the total contribution of the inelastic internal energy to the configurational subsystems.

Based on the equilibrium thermodynamics, the Cauchy stress can be calculated as $\sigma=2 \frac{1}{J} \mathbf{F} \frac{\partial \Psi}{\partial \mathbf{C}} \mathbf{F}^{\mathrm{T}}$, which gives,

$$
\sigma=\sum_{i}^{P} \underbrace{\frac{1}{J} \phi_{i} \frac{T_{e_{i}}}{T_{0}} \mu^{\text {eq }}\left(\overline{\mathbf{b}}-\frac{1}{3} \operatorname{tr}(\overline{\mathbf{b}})\right)}_{\mathbf{s}_{i}^{\text {eq }}}+\sum_{j}^{N} \underbrace{\frac{1}{J} \mu_{j}^{\text {neq }}\left(\overline{\mathbf{b}}_{j}^{\mathrm{e}}-\frac{1}{3} \operatorname{tr}\left(\overline{\mathbf{b}}_{j}^{\mathrm{e}}\right)\right)}_{\mathbf{s}_{j}^{\text {neq }}}+\underbrace{\frac{1}{2 J} \kappa\left(J^{2}-1\right)}_{p} \mathbf{1} .
$$

The configurational entropy can be calculated as $\eta_{i}^{c}=-\frac{\partial \Psi}{\partial T_{e_{i}}}$,

$$
\eta_{i}^{c}\left(T_{e_{i}}, \mathbf{C}\right)=\Delta c_{i} \ln \frac{T_{e_{i}}}{T_{2}}-\frac{\phi_{i}}{T_{0}} \frac{\mu^{e q}}{2}(\operatorname{tr}(\overline{\mathbf{C}})-3)
$$


We apply the following evolution equation for internal variable $\mathbf{b}_{j}^{\mathrm{e}}$ to satisfy the second law of thermodynamics [3032],

$$
-\frac{1}{2} \mathscr{L}_{v} \mathbf{b}_{j}^{e} \mathbf{b}_{j}^{\mathrm{e}-1}=\frac{1}{2 v_{j}} \mathbf{s}_{j}^{\mathrm{neq}},
$$

where $\mathscr{L}_{v} \mathbf{b}_{j}^{\mathrm{e}}=-2 \mathbf{F}_{j}^{\mathrm{e}} \mathbf{D}_{j}^{\mathrm{v}} \mathbf{F}_{j}^{\mathrm{e}^{\mathrm{T}}}$ is the Lie time derivative of the spatial tensor $\mathbf{b}_{j}^{\mathrm{e}}$ and $v_{j}$ is the viscosity. Thus, Equation (5) can also be written as, $\mathbf{D}_{j}^{\mathrm{v}}=\left(\mathbf{F}_{j}^{\mathrm{e}-1} \mathbf{s}_{j}^{\mathrm{neq}} \mathbf{F}_{j}^{\mathrm{e}}\right) /\left(2 v_{j}\right)$. The viscosity depends on the polymer configurational state which is described by Adam-Gibbs model [33], and the flow stress, described by an Eyring model [34],

$$
v_{j}\left(T, T_{e_{i}}, \mathbf{C}, s\right)=v_{j}^{r e f} \exp \left(\frac{B}{T \eta^{c}}\right) \frac{Q_{s} s}{T s_{y}}\left[\sinh \left(\frac{Q_{s} s}{T s_{y}}\right)\right]^{-1} .
$$

where $v_{j}^{\text {ref }}$ is the viscosity at a reference state, $s=\sqrt{\frac{1}{2} \sum_{j}^{N} \mathbf{s}_{j}^{\text {neq }}: \sum_{j}^{N} \mathbf{s}_{j}^{\text {neq }}}$ is the flow stress, $s_{y}$ is the yield strength, $Q_{\mathrm{s}}$ is activation parameter and $\eta^{c}=\sum_{i}^{P} \eta_{i}^{c}$ is the total configurational entropy. The stress relaxation time is defined as $\tau_{S_{j}}=v_{j} / \mu_{j}^{\text {neq }}$, which can be represented as,

$$
\tau_{S_{j}}\left(T, T_{e_{i}}, \mathbf{C}, s\right)=\tau_{S_{j}}^{r e f} \exp \left(\frac{B}{T \eta^{c}}\right) \frac{Q_{s} s}{T s_{y}}\left[\sinh \left(\frac{Q_{s} s}{T s_{y}}\right)\right]^{-1}
$$

where $\tau_{S_{j}}^{r e f}$ represents the reference stress relaxation time.

The following governing equation is derived from the energy balance for the configurational subsystem,

$$
\dot{T}_{e_{i}}=-\frac{T-T_{e_{i}}}{\tau_{R_{i}}}+\frac{a}{\Delta c} W^{i n t}+\frac{\mathscr{H}_{i}^{\mathrm{e}, \mathrm{c}}}{\Delta c \phi_{i}},
$$

where $\tau_{R_{i}}$ is structural relaxation time, $W^{\text {int }}$ is inelastic power and $\mathscr{H}_{i}^{\mathrm{e}, \mathrm{c}}$ is configurational contributions to the thermoelastic coupling term. The internal power can be represented as: $W^{i n t}=\left(\sum_{j}^{N} J \mathbf{s}_{j}^{\text {neq }}: \mathbf{s}_{j}^{\text {neq }}\right) / 2 v_{j}$ and the thermoelastic coupling term can be represented as $\mathscr{H}_{i}^{\mathrm{e}, \mathrm{c}}=J \mathbf{s}_{i}^{e q}: \mathbf{D}$. We have neglected in Equation (8) the divergence term of the effective temperature for simplicity [30]. The first term of the right side of Equation (8) represents physical aging, where the effective temperature of the configurational subsystem evolves towards the equilibrium temperature. This induces a more ordered structural state and decreases the chain mobility. The second term of the right side of Equation (8) represents the structural rejuvenation, where a fractional of internal plastic power is stored in the configurational subsystems, increasing the effective temperature. This increases the molecular mobility and induces strain softening.

The same Adam-Gibbs model is also applied to describe the structural relaxation time,

$$
\tau_{R_{i}}\left(T_{e_{i}}, \mathbf{C}\right)=\tau_{R_{i}}^{r e f} \exp \left(\frac{B}{T \eta^{c}}\right) .
$$

Equations (3) - (9) complete the effective temperature model.

\subsection{Internal variable approach}

To model the strain softening, Boyce et al. [9] proposed an evolution equation for the yield strength to represent a decrease in the energy barrier for viscous flow when the polymer is deformed. This approach has been applied successfully to describe experimental observations [29, 32, 35-37]. Here we extend this approach by adding a static recovery term commonly used for metals [18].

We use the following Helmholtz free energy density,

$$
\Psi=\frac{T}{T_{0}} \frac{\mu^{\mathrm{eq}}}{2}(\operatorname{tr}(\overline{\mathbf{C}})-3)+\sum_{j}^{N} \frac{\mu_{j}^{\mathrm{neq}}}{2}\left(\operatorname{tr}\left(\overline{\mathbf{C}}_{j}^{\mathrm{e}}\right)-3\right)+\frac{\kappa}{4}\left(J^{2}-2 \log J-1\right)+c_{g}\left(T-T_{0}\right)-c_{g} T \ln \frac{T}{T_{0}},
$$


and evaluate the Cauchy stress as,

$$
\sigma=\frac{1}{J} \frac{T}{T_{0}} \mu^{\text {eq }}\left(\overline{\mathbf{b}}-\frac{1}{3} \operatorname{tr}(\overline{\mathbf{b}})\right)+\sum_{j}^{N} \frac{1}{J} \mu_{j}^{\text {neq }}\left(\overline{\mathbf{b}}_{j}^{\mathrm{e}}-\frac{1}{3} \operatorname{tr}\left(\overline{\mathbf{b}}_{j}^{\mathrm{e}}\right)\right)+\frac{1}{2 J} \kappa\left(J^{2}-1\right) \mathbf{1} .
$$

The same evolution equation (5) for $\mathbf{b}_{j}^{\mathrm{e}}$ is used. We apply an Eyring stress-activation model with an Arrhenius temperature dependence for the viscosity,

$$
v_{j}(T, s)=v_{j}^{r e f} \exp \left(-\frac{F_{c}}{R T}\right) \frac{Q_{\mathrm{s}} s}{T s_{y}}\left[\sinh \left(\frac{Q_{\mathrm{s}} s}{T s_{y}}\right)\right]^{-1} .
$$

The $F_{c}$ is a thermal-activation energy for viscous flow.

To model strain softening and static recovery, we add a static recovery term to the evolution equation for the yield strength $s_{y}[9,29]$, where the recovery rate decreases exponentially with time,

$$
\dot{s}_{y}=h\left(1-\frac{s_{y}}{s_{\mathrm{yss}}}\right) \dot{\bar{\gamma}}^{v}+\frac{1}{\tau_{r}} \frac{Q_{r} s_{y}}{s_{y s s}}\left[\sinh \left(\frac{Q_{r} s_{y}}{s_{y s s}}\right)\right]^{-1}, \quad s_{y}(t=0)=s_{y 0} .
$$

The $h$ is the characteristic strain softening modulus, $s_{y 0}$ and $s_{\text {yss }}$ are the initial and steady state yield strength, $\dot{\bar{\gamma}}^{v}$ is the effective viscous strain rate, $\tau_{r}$ is the characteristic static recovery time and $Q_{r}$ is a pre-factor. This evolution equation is based on empirical observation of the main characteristics of the stress-strain curves of amorphous polymers [9]. Equation (5), (11), (12) and (13) complete the internal variable model. The explicit form of effective viscous strain rate $\dot{\bar{\gamma}}^{v}$ is shown in the Supplementary material. The methods used to determine the model parameters have been presented in detail in prior publications [29, 30]. The parameters and their values are listed in the Supplementary material.

\subsection{Comparison of the two models}

The effective temperature approach and internal variable approach share several similarities. When the configurational subsystems are in equilibrium with the kinetic subsystem $\left(T_{e_{i}}=T\right)$, the total Helmholtz free energy and stress response of the two models are identical. The two models also use the same viscous flow rule. Both models assume the strain softening is caused by a deformation-induced decrease in the viscosity. However, the underlying mechanisms are fundamentally different. For the effective temperature theory, a fraction of the inelastic power flows into the configurational subsystems to increase the configurational entropy and decrease in the viscosity through the AdamGibbs model in eq. (6). For the internal variable approach, the viscosity change caused by a decrease in the yield stress of the Eyring flow rule with the viscous strain rate. Physical aging occurs through an increase in the yield strength with time. In the effective temperature approach, physical aging is caused by the evolution of effective temperature towards the equilibrium, which decreases the configurational entropy and increase the viscosity.

\section{Results}

We applied both models to evaluate the temperature and rate-dependent and temperature-dependence in uniaxial compression. In general, both models were able to reproduce the dependence of the yield stress, post-yield stress drop and draw stress on temperature and strain rate (Figure 3 and 4). The experimental and simulation results of uniaxial compression response of polymers deformed at strain rate 0.01/s are shown in Figure 5. For clarity, we only plotted the results for $30^{\circ} \mathrm{C}$ and $10^{\circ} \mathrm{C}$. To quantitatively compare the experimental and simulation results, the yield stress and steady-state draw stress determined at $20 \%$ strain are plotted in Fig. 6. Both the internal variable model and effective temperature model showed good agreement with experiments at all strain rates and nearly all temperatures. However, the effective temperature theory significantly under-predicted the yield stress and draw stress at $0^{\circ} \mathrm{C}$. In previous work, we showed that the yield strength is sensitive to the structural relaxation spectrum, which is difficult to measure 
at low temperatures [29]. The structural relaxation spectrum was measured from the time-dependent thermal strain response to abrupt changes in temperature near $T_{\mathrm{g}}$. The test used a narrow range of temperature from $T_{\mathrm{g}}-8^{\circ} \mathrm{C}\left(28^{\circ} \mathrm{C}\right)$ to $T_{\mathrm{g}}+8^{\circ} \mathrm{C}$ to calibrate the structural relaxation spectrum, because the thermal deformation at lower temperatures was smaller than the measurement accuracy. The above results also showed that the effective temperature approach predicts that the stress continued to decrease after the post-yield stress drop before attaining a steady-state near 30\% strain. The development of a steady-state draw stress occurred at larger strains with increasing strain rate and lower temperatures. In contrast, the stress response of the internal variable model increased again shortly after the post yield stress drop, which was more consistent with experimental observation. We used a constant value for the parameter $a$ that describes the contribution of the nonequilibrium internal energy to the configurational subsystems. Consequently, plastic deformation continued to rejuvenate the stress response until it could be balanced by the effects of aging. The process of mechanical rejuvenation can be curtailed at a smaller strain by assuming a decreasing function of $a$ with the effective temperature.

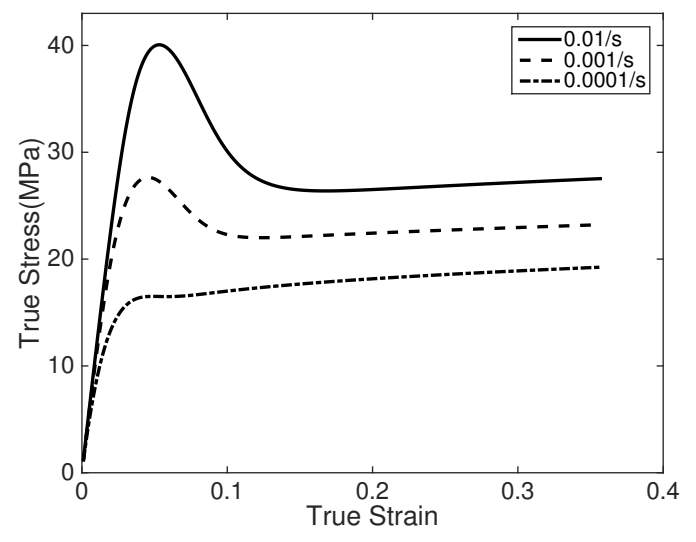

(a)

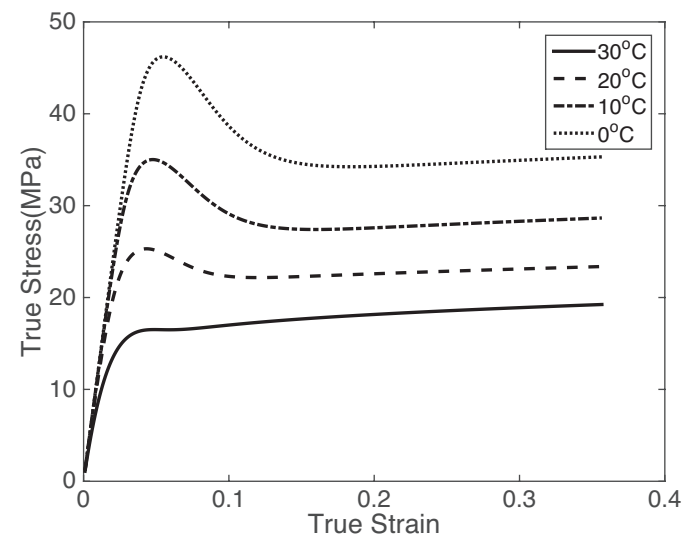

(b)

Figure 3: Simulations results of uniaxial compression response of acrylate-based copolymer using the internal variable model (a) at $30^{\circ} \mathrm{C}(\mathrm{b})$ at $0.0001 / \mathrm{s}$

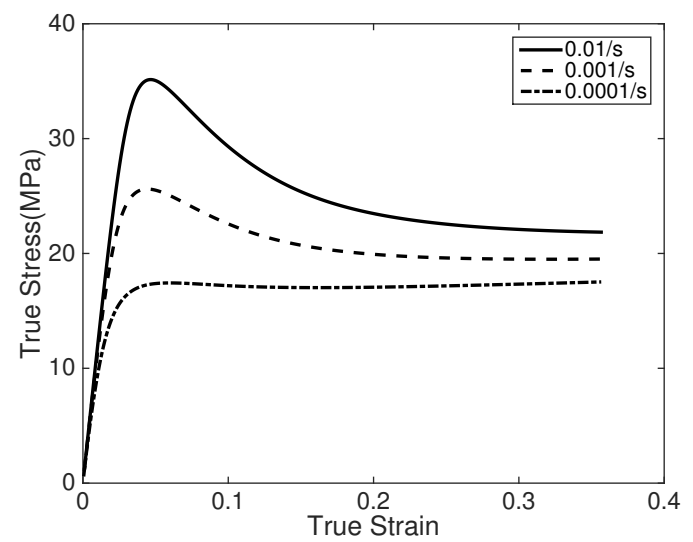

(a)

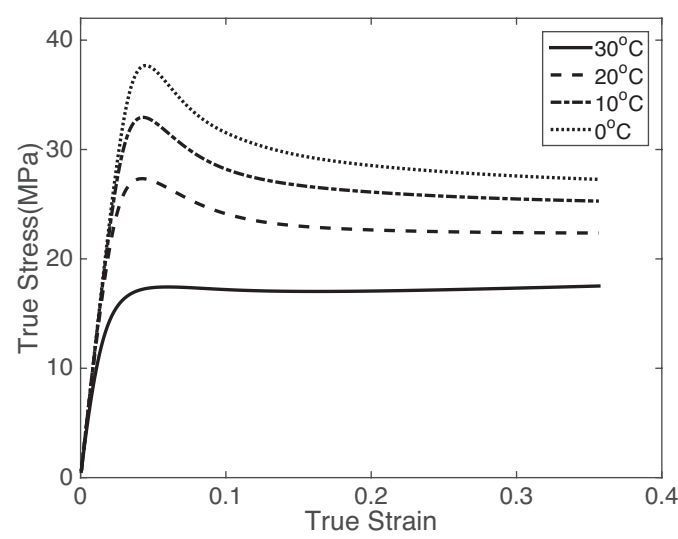

(b)

Figure 4: Simulation results of uniaxial compression response of acrylate-based copolymer using the effective temperature model (a) at $30^{\circ} \mathrm{C}(\mathrm{b})$ at $0.0001 / \mathrm{s}$

We next applied the two models to simulate the effect of physical aging and mechanical pre-deformation on the stress response (Figure 7 and 8). A comparison between experiments and model predictions of the stress response for different annealing time is shown in Figure 9. Both models were able to capture the increase in the yield strength and 


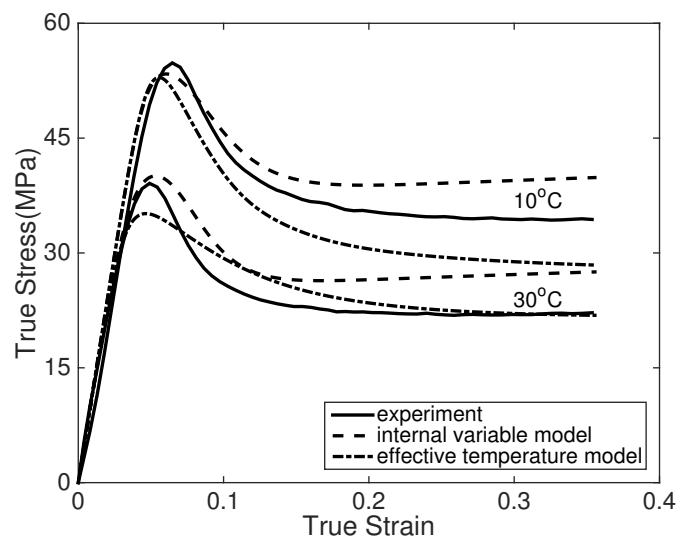

Figure 5: Comparison of experimental and simulation results of uniaxial compression response of acrylate-based copolymer at different temperatures and strain rate $0.01 / \mathrm{s}$

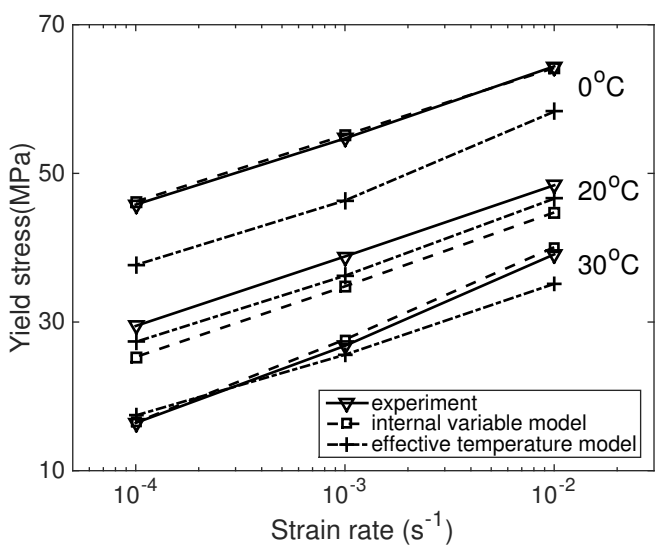

(a)

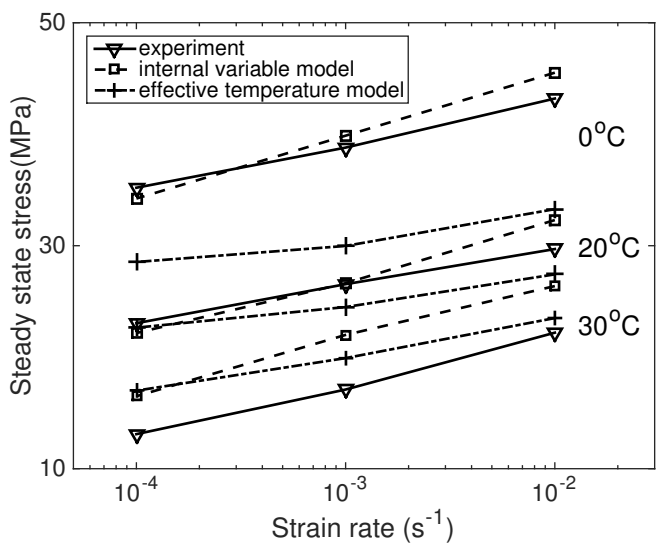

(b)

Figure 6: Comparison of experimental measured and simulation predicted a) yield strength and b) steady state stress of specimens deformed at different temperatures and strain rates. 
post-yield stress drop with the annealing time, the appearance of the steady-state draw stress, and effects of renewed physical aging. However, the internal variable model did not accurately predict the strain recovery during the annealing period between first and second compression loading. The internal variable model predicted negligible strain recovery for the 1 minute annealing time and significantly larger strain recovery for longer annealing times. In contrast the effective temperature model accurately predicted the recovered strains measured in experiments for different aging times. The relaxation time is dependent on the nonequilibrium state in the effective temperature model. The effective temperature model is more mobile with a smaller relaxation time, thus a large strain recovery is observed (around $1 \%$ ) in the initial minutes after recovery. However, the material undergoes physical aging immediately after unloading as the effective temperatures evolves to the equilibrium temperature. This causes the relaxation time to increase and the recovery rate to decrease with time. The internal variable model does not capture the coupling between the nonequilibrium structure and relaxation time. Thus the recovery rate continued to increase with time.

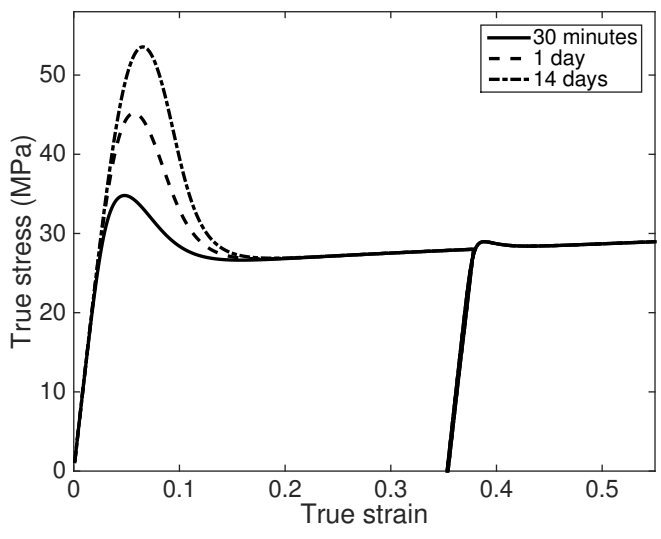

(a)

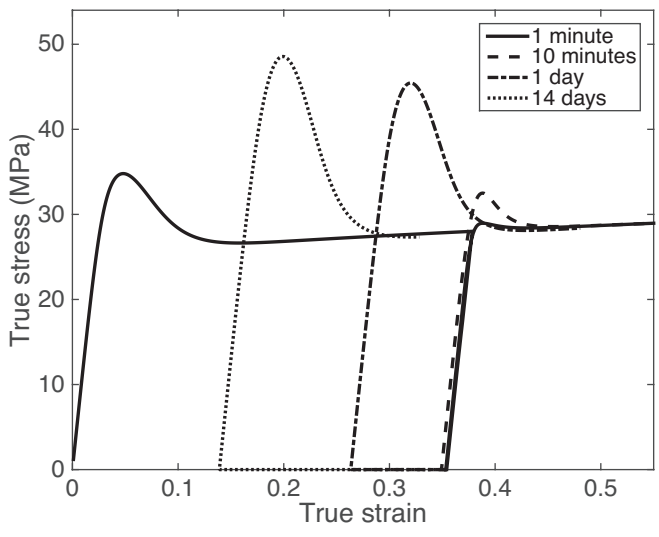

(b)

Figure 7: Simulation results of uniaxial compression response of acrylate-based copolymer for two load-unload cycles at $0.001 / \mathrm{s}$ strain rate using the internal variable model (a) different annealing times prior to the first cycle and (b) different annealing time prior to the second cycle

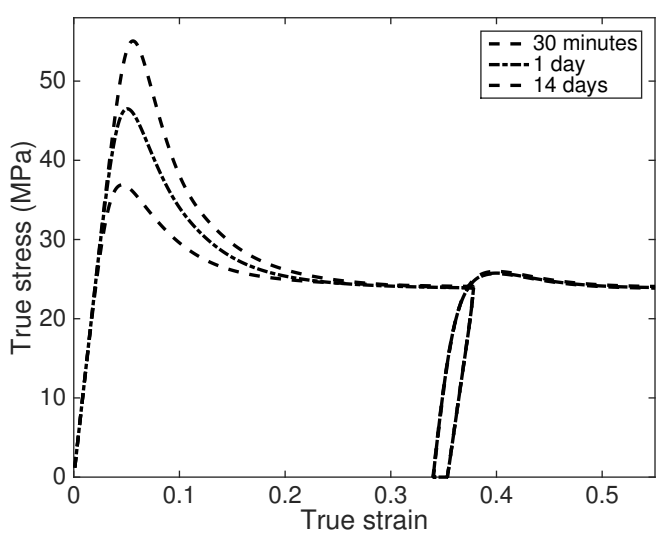

(a)

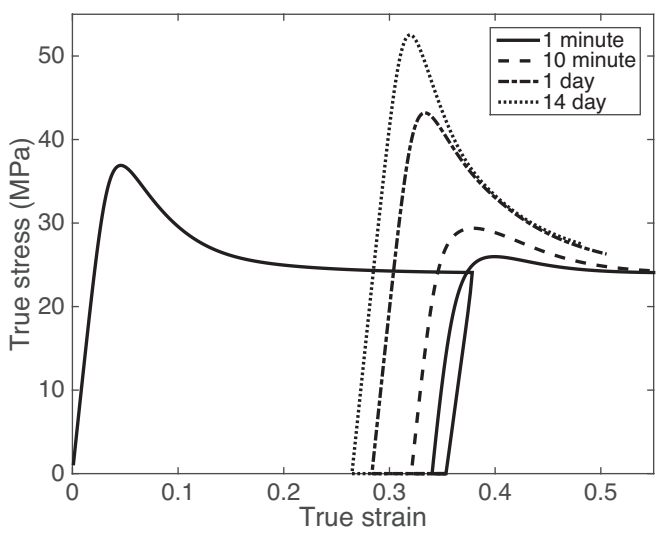

(b)

Figure 8: Simulation results of uniaxial compression response of acrylate-based copolymer for two load-unload cycles at $0.001 / \mathrm{s}$ strain rate using the effective temperature model (a) different annealing times prior to the first cycle and (b) different annealing time prior to the second cycle 


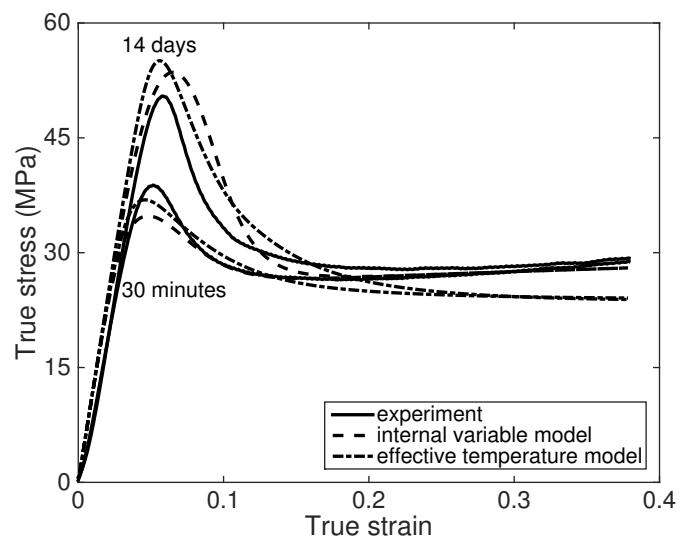

Figure 9: Comparison of experimental and simulation results of uniaxial compression response of acrylate-based copolymer with different aging time

\section{Conclusions}

In summary, we developed a thermomechanical theory for the nonequilibrium behavior of glassy polymers that couples plastic deformation and structural evolution. The theory is based on the effective temperature thermodynamic framework, described by Kamrin and Bouchbinder [22] and Xiao and Nguyen [30], that introduces a distribution of effective temperatures to describe the nonequilibrium structure. The governing equation for the effective temperature is derived from the first law and includes the effects of physical aging (structural relaxation) and mechanical rejuvenation, where a portion of the plastic power drives the nonequilibrium structure towards a more disordered state. The nonequilibrium structure affects the plastic flow through a modified Adam-Gibbs model [33]. We showed that the effective temperature model was able quantitatively predict the effects of physical aging and cold work on the yield and post-yield softening behaviors measured in experiments. We compared the predictions of the effective temperature model with the internal variable model of Boyce et al [9]. We added the a static recovery term to the evolution of the yield strength to describe physical aging. Both models were able to capture the effect of strain rate, temperature, physical aging and mechanical rejuvenation. However, the internal variable model could not capture the strain recovery of the unloaded material.

Compared to the internal variable softening model, the effective temperature approach provides a more fundamental description of the nonequilibrium structure and can be applied to describe a wide array of nonequilibrium behaviors. We showed in Xiao et al [29] and Xiao and Nguyen [30] that the Xiao and Nguyen model describes the glass transition in the stress response, thermal expansion response and enthalpy response. The effective temperature model shows the possibility of providing a unified model with a minimum set of parameters for a wide range of nonequilibrium behaviors, which are typically treated as separate phenomena in current modeling approaches.

\section{Acknowledgements}

R. Xiao acknowledges the funding support from the National Natural Science Foundation of China (Grant No. 11502068). The authors also gratefully acknowledge the funding support from the National Science Foundation ( CMMI-1130358).

\section{References}

[1] L. Govaert, P. Timmermans, W. Brekelmans, The influence of intrinsic strain softening on strain localization in polycarbonate: modeling and experimental validation, Journal of Engineering Materials and Technology 122 (2) 
(2000) 177-185.

[2] L. Anand, O. Aslan, S. A. Chester, A large-deformation gradient theory for elastic-plastic materials: strain softening and regularization of shear bands, International Journal of Plasticity 30 (2012) 116-143.

[3] C. GŚell and G. B. McKenna, Influence of physical ageing on the yield response of model dgeba/poly(propylene oxide) epoxy glasses, Polymer 33 (1992) 2103 - 2113

[4] L. C. E. Struik, On the rejuvenation of physically aged polymers by mechanical deformation, Polymer 38 (1997) $4053-4057$.

[5] H. E. Meijer, L. E. Govaert, Mechanical performance of polymer systems: the relation between structure and properties, Progress in polymer science 30 (8) (2005) 915-938.

[6] O. Hasan, M. C. Boyce, Energy storage during inelastic deformation of glassy polymers, Polymer 34 (24) (1993) 5085-5092.

[7] Z. Yang, J. Wang, Coupled annealing temperature and layer thickness effect on strengthening mechanisms of ti/ni multilayer thin films, Journal of the Mechanics and Physics of Solids 88 (2016) 72-82.

[8] J. Rottler, M. O. Robbins, Unified description of aging and rate effects in yield of glassy solids, Physical review letters 95 (22) (2005) 225504.

[9] M. C. Boyce, D. M. Parks, A. S. Argon, Large inelastic deformation of glassy polymers. part i: rate dependent constitutive model, Mechanics of Materials 7 (1) (1988) 15-33.

[10] O. Hasan, M. Boyce, A constitutive model for the nonlinear viscoelastic viscoplastic behavior of glassy polymers, Polymer Engineering \& Science 35 (4) (1995) 331-344.

[11] E. Klompen, T. Engels, L. Govaert, H. Meijer, Modeling of the postyield response of glassy polymers: influence of thermomechanical history, Macromolecules 38 (16) (2005) 6997-7008.

[12] C. Buckley, P. Dooling, J. Harding, C. Ruiz, Deformation of thermosetting resins at impact rates of strain. part 2: constitutive model with rejuvenation, Journal of the Mechanics and Physics of Solids 52 (10) (2004) 2355-2377.

[13] L. Anand, N. M. Ames, On modeling the micro-indentation response of amorphous polymer, Int. J. Plas. 22 (2006) 1123-1170.

[14] K. Chen, K. S. Schweizer, Theory of aging, rejuvenation, and the nonequilibrium steady state in deformed polymer glasses, Physical Review E 82 (4) (2010) 041804.

[15] K. Chen, K. S. Schweizer, Theory of yielding, strain softening, and steady plastic flow in polymer glasses under constant strain rate deformation, Macromolecules 44 (10) (2011) 3988-4000.

[16] M. Jiang, G. Wilde, L. Dai, Origin of stress overshoot in amorphous solids, Mechanics of Materials 81 (2015) $72-83$.

[17] A. Q. Tool, Viscosity and extraordinary heat effects in glass., J. Am. Ceram. Soc. 29 (1946) $240-253$.

[18] J. Chaboche, A review of some plasticity and viscoplasticity constitutive theories, International Journal of Plasticity 24 (10) (2008) 1642-1693.

[19] T. M. Nieuwenhuizen, Thermodynamics of the glassy state: Effective temperatureas an additional system parameter, Phys. Rev. Lett. 80 (1998) 5580-5583.

[20] J. Langer, Dynamics of shear-transformation zones in amorphous plasticity: Formulation in terms of an effective disorder temperature, Physical Review E 70 (4) (2004) 041502.

[21] E. Bouchbinder, J. S. Langer, Nonequilibrium thermodynamics of driven amorphous materials. ii. effectivetemperature theory, Phys. Rev. E. 80 (2009) 031132-1-094505-7. 
[22] K. Kamrin, E. Bouchbinder, Two-temperature continuum thermomechanics of deforming amorphous solids, J. Mech. Phys. Solids 73 (2014) 269-288.

[23] H. A. Makse, J. Kurchan, Testing the thermodynamic approach to granular matter with a numerical model of a decisive experiment, Nature 415 (6872) (2002) 614-617.

[24] I. K. Ono, C. S. OHern, D. Durian, S. A. Langer, A. J. Liu, S. R. Nagel, Effective temperatures of a driven system near jamming, Physical review letters 89 (9) (2002) 095703.

[25] M. L. Falk, J. Langer, Deformation and failure of amorphous, solidlike materials, Annual Review of Condensed Matter Physics 2 (2011) 353-373.

[26] P. Chadwick, Thermo-mechanics of rubberlike materials, Philosophical Transactions of the Royal Society of London A: Mathematical, Physical and Engineering Sciences 276 (1260) (1974) 371-403.

[27] O. Hasan, M. Boyce, X. Li, S. Berko, An investigation of the yield and postyield behavior and corresponding structure of poly (methyl methacrylate), Journal of polymer science part B: polymer physics 31 (2) (1993) 185197.

[28] D. Rittel, On the conversion of plastic work to heat during high strain rate deformation of glassy polymers, Mechanics of Materials 31 (2) (1999) 131-139.

[29] R. Xiao, J. Choi, N. Lakhera, C. Yakacki, C. Frick, T. Nguyen, Modeling the glass transition of amorphous networks for shape-memory behavior, Journal of the Mechanics and Physics of Solids 61 (7) (2013) 1612-1635.

[30] R. Xiao, T. D. Nguyen, An effective temperature theory for the nonequilibrium behavior of amorphous polymers, Journal of the Mechanics and Physics of Solids 82 (2015) 62-81.

[31] S. Reese, S. Govindjee, A theory of finite viscoelasticity and numerical aspects, Int. J. Solids Struct. 35 (1998) 3455-82.

[32] T. Nguyen, H. J. Qi, F. Castro, K. N. Long, A thermoviscoelastic model for amorphous shape memory polymers: Incorporating structural and stress relaxation, J. Mech. Phys. Solids 56 (2008) 2792-2814.

[33] G. Adam, J. H. Gibbs, On the temperature dependence of cooperative relaxation properties in the glass-forming liquids, J. Chem. Phys. 43 (1965) 139-146.

[34] H. Eyring, Viscosity, Plasticity, and Diffusion as Examples of Absolute Reaction Rates, J. Chem. Phys. 4 (1936) 283-291.

[35] E. M. Arruda, M. C. Boyce, R. Jayachandran, Effects of strain rate, temperature and thermomechanical coupling on the finite strain deformation of glassy polymers, Mechanics of Materials 19 (2) (1995) 193-212.

[36] J.-L. Bouvard, D. K. Francis, M. A. Tschopp, E. Marin, D. Bammann, M. Horstemeyer, An internal state variable material model for predicting the time, thermomechanical, and stress state dependence of amorphous glassy polymers under large deformation, International Journal of Plasticity 42 (2013) 168-193.

[37] P. Yu, X. Yao, Q. Han, S. Zang, Y. Gu, A visco-elastoplastic constitutive model for large deformation response of polycarbonate over a wide range of strain rates and temperatures, Polymer 55 (25) (2014) 6577-6593. 